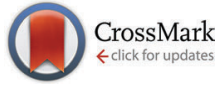

Cite this: Chem. Commun., 2015, 51, 2036

Received 29th October 2014,

Accepted 17th November 2014

DOI: $10.1039 / \mathrm{c} 4 \mathrm{cc} 08541 \mathrm{~h}$

www.rsc.org/chemcomm

\section{Dual-responsive nanoparticles that aggregate under the simultaneous action of light and $\mathrm{CO}_{2} \dagger$}

\author{
Ji-Woong Lee and Rafal Klajn*
}

Metallic nanoparticles co-functionalised with monolayers of UV- and $\mathrm{CO}_{2}$-sensitive ligands were prepared and shown to respond to these two types of stimuli reversibly and in an orthogonal fashion. The composition of the coating could be tailored to yield nanoparticles capable of aggregating exclusively when both $\mathrm{UV}$ and $\mathrm{CO}_{2}$ were applied at the same time, analogously to the behaviour of an AND logic gate.

Stimuli-responsive nanomaterials ${ }^{1}$ that self-assemble in a reversible fashion are important because of their emerging applications in diverse fields, such as switchable catalysis, ${ }^{2,3}$ water purification, ${ }^{4}$ detection of analytes, ${ }^{5}$ and time-sensitive information storage. ${ }^{6}$ Accordingly, a variety of nanoparticles (NPs) have been engineered to aggregate in response to different external stimuli, such as a magnetic field, ${ }^{7} \mathrm{pH},{ }^{8,9}$ or chemical fuel. ${ }^{10}$ For example, dynamic aggregation of metallic NPs was accomplished by reversibly photoswitching the electronic states and geometries of surface-bound chromophores. ${ }^{11-14}$ New applications could arise, however, for NPs that respond to two (or more) different external stimuli at once, such as the recently reported dual-responsive NPs that aggregate when either a magnetic field or light is applied. ${ }^{15-18}$ Whereas such dual-responsiveness is, in principle, relatively easy to "encode" in the NPs, it is more challenging to design systems capable of aggregating only when two types of external stimuli are applied at the same time. ${ }^{19,20}$ Here, we describe the first system exhibiting such behaviour with light and carbon dioxide as the two "inputs".

Carbon dioxide is a biocompatible, naturally abundant gas that has several advantages over other chemical stimuli: (i) facile reactivity with various nucleophilic Lewis base functional groups such as amines, NHCs (N-heterocyclic carbenes), or guanidines, (ii) easy removal from the above complexes by mild heating, sonication, or purging with an inert gas, (iii) no by-product formation through the reversible complex formation, and (iv) potential applicability to reversible $\mathrm{CO}_{2}$ capture and/or transformations. ${ }^{21-24}$ In addition,

Department of Organic Chemistry, Weizmann Institute of Science, 76100 Rehovot, Israel.E-mail: rafal.klajn@weizmann.ac.il

$\dagger$ Electronic supplementary information (ESI) available: Synthesis and characterisation of ligand 3; synthesis and functionalisation of gold nanoparticles; and control experiments. See DOI: $10.1039 / \mathrm{c} 4 \mathrm{cc} 08541 \mathrm{~h}$ nanomaterials that aggregate reversibly in the presence of $\mathrm{CO}_{2}$ are potentially important as possible anti-cancer therapeutics (taking advantage of the increased production of $\mathrm{CO}_{2}$ in cancer cells ${ }^{25,26}$ ). Although many $\mathrm{CO}_{2}$-sensitive materials, ${ }^{27}$ in particular, organic polymers, ${ }^{28,29}$ have been reported, the application of amine-functionalised metallic NPs as $\mathrm{CO}_{2}$-responsive materials remains unknown. Here, we presumed that control of the NP surface polarity by using fine-tuned amounts of photo- and $\mathrm{CO}_{2}$-sensitive ligands could induce selective and reversible aggregation of NPs with two orthogonal stimuli (Fig. 1).

It is well established that mixtures of $\omega$-functionalised long-chain alkanethiols can form mixed self-assembled monolayers (mSAMs) on the surfaces of metallic $\mathrm{NPS}^{30}$ (here, we focused on gold NPs). We commenced our study with AuNPs functionalised with mSAMs comprising an azobenzene-terminated thiol (AzoSH) and a "background" ligand, 1-dodecanethiol $\left(\mathbf{C}_{\mathbf{1 2}} \mathbf{S H}\right)$ (Fig. 2a). As the UV-Vis spectra in Fig. 2c show, NPs decorated with critical (>50\%) amounts of AzoSH aggregated in toluene upon exposure to long-wave $(\lambda=365 \mathrm{~nm}) \mathrm{UV}$ light, ${ }^{31}$ whereas they exhibited no detectable response to $\mathrm{CO}_{2}$ (confirmed by dynamic light scattering (DLS) measurements; see ESI, $\dagger$ Fig. S2 in Section S5). Furthermore, we observed that the trans-to-cis photoisomerisation of AzoSH did not affect the affinity of these NPs towards $\mathrm{CO}_{2}$ (Fig. S2, ESI $\dagger$ ). Therefore, we concluded that those AuNPs
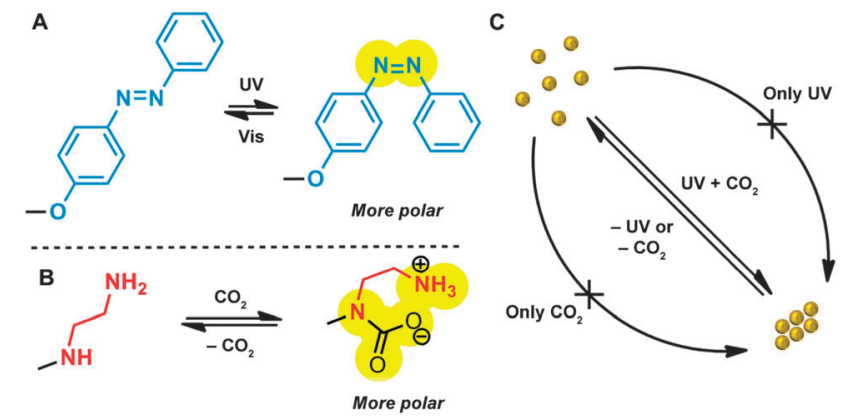

Fig. 1 (A) Reversible isomerisation of a photoswitchable ligand. (B) Reversible $\mathrm{CO}_{2}$ complexation by a diamine ligand. (C) A dual-stimuli-responsive AND logic gate. 
A

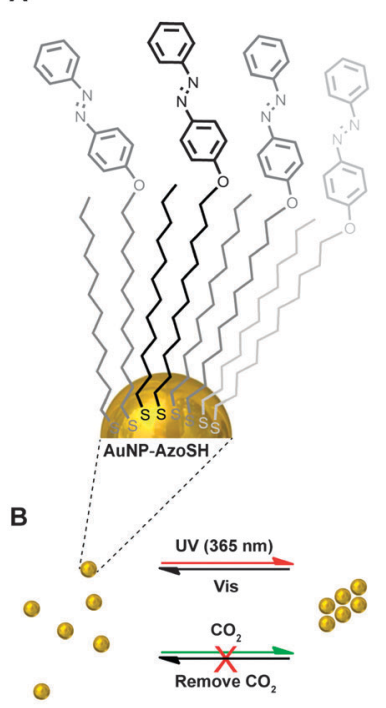

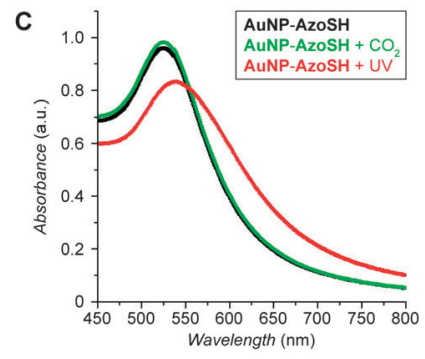

D

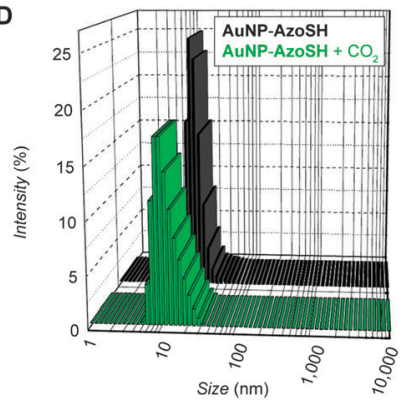

Fig. 2 Light-sensitive ligand (AzoSH)-decorated gold nanoparticles (AuNP_ AzoSH) (A) aggregate in response to light while showing no response to $\mathrm{CO}_{2}$ (B). UV-Vis absorption spectra (C) and DLS (D) of toluene solutions of AuNPAzoSH before and after exposure to UV and bubbling $\mathrm{CO}_{2}$.

bearing only the AzoSH and $\mathbf{C}_{\mathbf{1 2}} \mathbf{S H}$ ligands are inert towards the $\mathrm{CO}_{2}$ stimulus under these conditions.

The lack of general knowledge on the construction of $\mathrm{CO}_{2}$ sensitive metal-nanoparticle surfaces encouraged us to investigate the synthesis and application of thiol-terminated amine ligands (Fig. 1b). ${ }^{32}$ Based on previous literature reports, ${ }^{33-36}$ we presumed that ethylenediamine derivatives are capable of $\mathrm{CO}_{2}$ complexation under low-pressure conditions. To verify the effect of $\mathrm{CO}_{2}$ binding on the solubility in toluene, we synthesised and studied the behaviour of a model compound, $N$-decylethylenediamine. As detailed in the $\mathrm{ESI}_{\dagger}$ (Section S3), this diamine was readily soluble in toluene; however, rapid precipitation commenced upon bubbling $\mathrm{CO}_{2}$ through the solution (Fig. S1, ESI $\dagger$ ). The process was reversible: the addition of an inert gas (e.g. $\mathrm{N}_{2}$ ) caused the precipitate to redissolve. Encouraged by these results, we then proceeded to synthesise AuNPs decorated with the diamine moieties. Although diamine-functionalised NPs were previously prepared by reacting 11-bromoundecane-1-thiol (2)-decorated NPs with 1,2-ethylenediamine, we began our investigation by preparing a well-defined thiol bearing the diamine moiety. As shown in Scheme 1, the

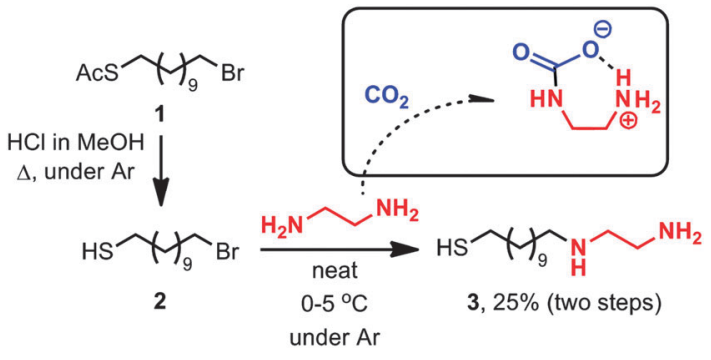

Scheme 1 Synthesis of diamine ligand 3.

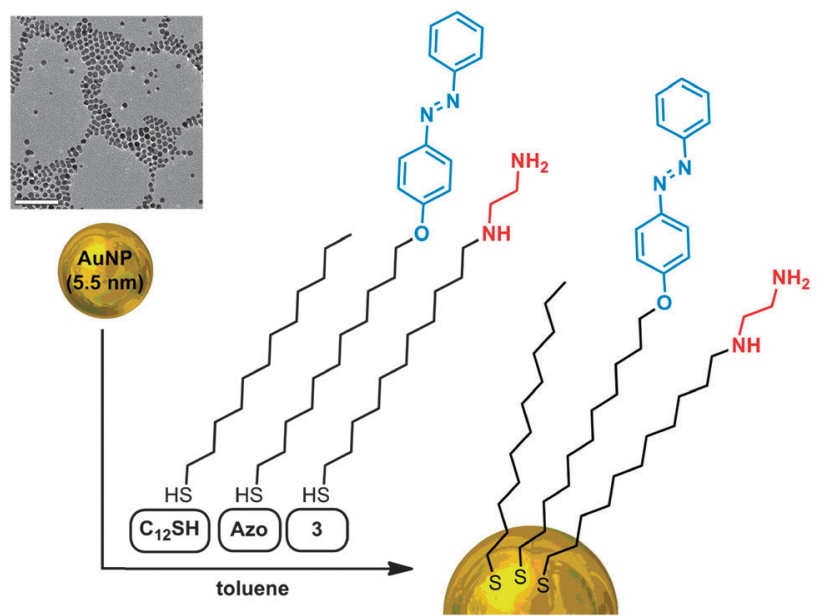

Fig. 3 Preparation of dual-responsive AuNPs functionalised with an mSAM comprising $\mathrm{C}_{12} \mathrm{SH}$, AzoSH and diamine 3 (the scale bar in the TEM image is $50 \mathrm{~nm}$ ).

synthesis of diamine 3 was straightforward, starting with a known material $\mathbf{1 .}^{37-39}$

With the diamine-functionalised thiolate ligand 3 in hand, we prepared a family of dual stimuli-responsive AuNPs by varying the ratio of the two responsive ligands (AzoSH and diamine 3) and the background ligand 1-dodecanethiol $\left(\mathbf{C}_{\mathbf{1 2}} \mathbf{S H}\right)$, as shown in Fig. 3. Although 3 could potentially bind to AuNPs via both the mercapto and the amine functionalities, the latter scenario is unlikely given the $c a$. two-orders-of-magnitude higher affinity of $\mathrm{Au}$ to thiols as compared to amines. ${ }^{12}$ In addition, binding to $\mathrm{Au}$ via the amine functionalities would cause NP crosslinking and irreversible aggregation, which we did not observe. ${ }^{40}$

Owing to the high polarity and low toluene solubility of the diamine ligand $\mathbf{3}$, the ligand exchange reaction was conducted under dilute ( $c a .10$ times) conditions, compared with typical ligand exchange reaction conditions. ${ }^{41}$ Moreover, 3 was always used after drying under vacuum at $40{ }^{\circ} \mathrm{C}$ to ensure that it was not complexed with $\mathrm{CO}_{2}$. The functionalised AuNPs were precipitated with methanol $(1: 1 \mathrm{v} / \mathrm{v})$ in the presence of didodecyldimethylammonium bromide ( $0.5 \mathrm{mg}$ per $0.716 \mathrm{mg}$ of $\mathrm{Au}$ ), washed extensively with methanol to remove any unbound molecules, dried under vacuum and kept in an inert atmosphere prior to use. A good indication of the presence of 3 in the functionalised AuNPs is their good solubility in polar solvents (e.g., ethanol, methanol and even water), which confirms a successful ligand-exchange reaction (for comparison, AuNP-AzoSH is soluble only in nonpolar solvents such as toluene, chloroform, or dichloromethane). The functionalisation procedure did not affect the size or size distribution of the NPs, whose monodispersity was confirmed by transmission electron microscopy (Fig. 5a).

Fine-tuning of the ratio of the three ligands was critical for obtaining NPs exhibiting the behaviour shown in Fig. 1c (in this context, it is worth mentioning that the previously adapted $^{32,34}$ on-nanoparticle reaction between ethylenediamine and 2-functionalised AuNPs would hardly be applicable). We found that when the molar percentage of 3 in the NPs exceeded $25 \%$, the particles were insoluble in toluene due to their high 


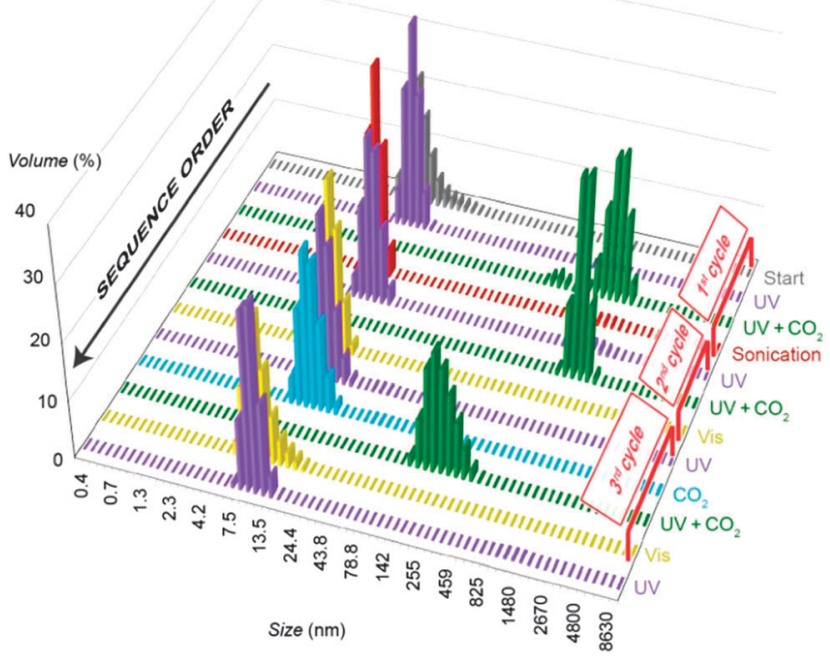

Fig. 4 Reversible, dual-stimuli-responsive clustering of AuNPs studied by dynamic light scattering.

surface polarity. These aggregated NPs could not be redissolved using visible light, sonication, or by purging with $\mathrm{N}_{2}$ (to remove any possibly complexed $\mathrm{CO}_{2}$ ). Therefore, the amount of diamine 3 was fixed at $25 \%$ throughout the optimisation of the $m \mathrm{SAM}$ composition. Having screened the ratio of the remaining two ligands (AzoSH and $\mathbf{C}_{\mathbf{1 2}} \mathbf{S H}$ ) from 1:2 to 2:1, we found that NPs containing 33-35 mol\% of AzoSH within the $m$ SAM exhibited the behaviour shown in Fig. 1c. According to DLS, these NPs had hydrodynamic diameters of 7-10 $\mathrm{nm}$ in toluene, and did not aggregate upon either only UV irradiation or $\mathrm{CO}_{2}$ treatment (bubbling into the solution) (purple and blue bars, respectively, in Fig. 4). Only when both stimuli were applied at once (4 min of UV irradiation during $\mathrm{CO}_{2}$ bubbling) did the NPs rapidly assemble into aggregates of $800-1000 \mathrm{~nm}$ in size, as shown by DLS (green bars in Fig. 4). The presence of aggregated NPs was also evident from TEM and spectroscopic analysis (Fig. $5 \mathrm{~b}$ and c). Interestingly, these aggregates were stable in a closed vial placed in the dark for more than 30 minutes.

Further demonstration of the AND gate-like behaviour was provided by removing one of the two stimuli. For example, when a solution of aggregated NPs was sonicated, facile disaggregation was observed even under constant UV irradiation (red bars in Fig. 4; $c a .10 \mathrm{~nm}$ ). The resulting NPs did not respond to only $\mathrm{CO}_{2}$ or UV, but they could again be quickly aggregated by applying UV and $\mathrm{CO}_{2}$ simultaneously (second-cycle green bars in Fig. 4 and the TEM image in Fig. 5b). Interestingly, our NPs also assembled as a result of $\mathrm{CO}_{2}$ bubbling ( $4 \mathrm{~min}$ ), followed by exposure to UV light, or via the opposite sequence, whereby UV-irradiated NPs were treated with $\mathrm{CO}_{2}$ in the dark: these results indicate that both (i) cis-azobenzene and (ii) the $\mathrm{CO}_{2}$-diamine complex are relatively stable, (i) in the dark, and (ii) unless other stimulants (such as sonication, heating or inert gas bubbling) are applied.

Thus assembled AuNPs readily deaggregated under ambient light (5-6 minutes) or upon exposure to visible light (fluorescent bulb, $<30$ seconds), and consequently, additional assembly-disassembly

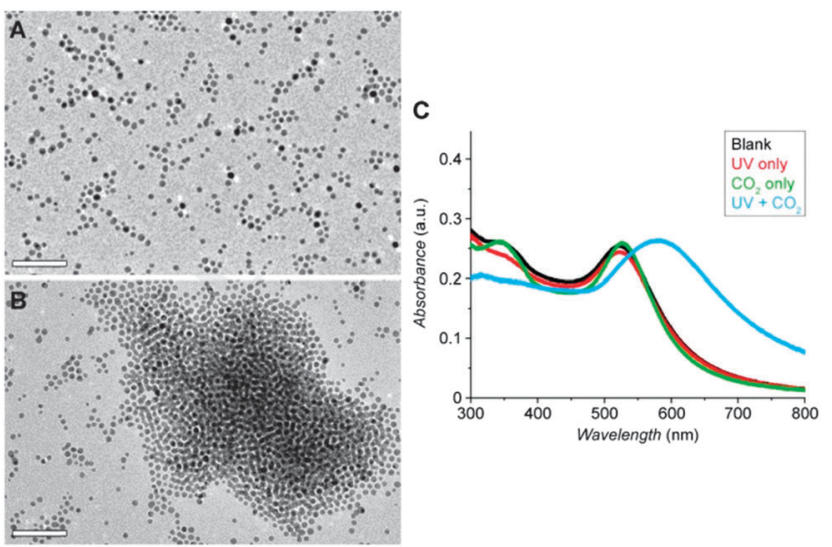

Fig. 5 (A) TEM images of AuNPs before aggregation. (B) The same NPs after applying two stimuli $\left(\mathrm{CO}_{2}\right.$ and UV) at once. (C) UV spectra of AuNPs with only one stimulus (red and green) and both stimuli at once (blue). The scale bars in TEM images are $50 \mathrm{~nm}$.

cycles could be performed. Overall, we concluded that this study represents the first example of $\mathrm{CO}_{2}$ - and light-responsive nanoparticles, whose behaviour constitutes an AND logic gate. Noteworthy, NPs having higher surface concentrations of AzoSH did respond to UV light by assembling into aggregates, which could aggregate further upon the delivery of $\mathrm{CO}_{2}$ (ESI, $\dagger$ Fig. S6C). In other words, only upon pre-exposure to UV light were these specific NPs responsive to $\mathrm{CO}_{2}$.

We prepared gold nanoparticles co-functionalised with azobenzene- and diamine-terminated ligands, which rendered the particles responsive to UV irradiation and $\mathrm{CO}_{2}$, respectively. Exposure to UV and $\mathrm{CO}_{2}$ led to a stepwise increase in the nanoparticle surface polarity, triggering aggregation in a nonpolar medium (toluene). ${ }^{42}$ The composition of the mixed monolayer of ligands could be tuned in order to afford NPs capable of aggregating only when both types of stimuli are applied at once. Both of these stimuli could easily be turned off/removed without the generation of any by-products/chemical waste, after which the assembly-disassembly cycles could be repeated. The aggregates exist only when both light and $\mathrm{CO}_{2}$ are applied simultaneously. An important future direction concerning these and other man-made dynamically aggregating materials is to study/engineer their emergent behaviours under non-equilibrium conditions.

\section{Notes and references}

1 Intelligent Stimuli-Responsive Materials. From Well-Defined Nanostructures to Applications, ed. Q. Li, John Wiley and Sons, Hoboken, 2013. 2 V. Polshettiwar, R. Luque, A. Fihri, H. B. Zhu, M. Bouhrara and J. M. Bassett, Chem. Rev., 2011, 111, 3036-3075.

3 Y. H. Zhu, L. P. Stubbs, F. Ho, R. Z. Liu, C. P. Ship, J. A. Maguire and N. S. Hosmane, ChemCatChem, 2010, 2, 365-374.

4 C. T. Yavuz, J. T. Mayo, W. W. Yu, A. Prakash, J. C. Falkner, S. Yean, L. L. Cong, H. J. Shipley, A. Kan, M. Tomson, D. Natelson and V. L. Colvin, Science, 2006, 314, 964-967.

5 R. Elghanian, J. J. Storhoff, R. C. Mucic, R. L. Letsinger and C. A. Mirkin, Science, 1997, 277, 1078-1081.

6 R. Klajn, P. J. Wesson, K. J. M. Bishop and B. A. Grzybowski, Angew. Chem., Int. Ed., 2009, 48, 7035-7039.

7 G. Singh, H. Chan, A. Baskin, E. Gelman, N. Repnin, P. Kral and R. Klajn, Science, 2014, 345, 1149-1153. 
8 R. Sardar, N. S. Bjorge and J. S. Shumaker-Parry, Macromolecules, 2008, 41, 4347-4352.

9 J. Simard, C. Briggs, A. K. Boal and V. M. Rotello, Chem. Commun., 2000, 1943-1944.

10 J. Boekhoven, A. M. Brizard, K. N. K. Kowlgi, G. J. M. Koper, R. Eelkema and J. H. van Esch, Angew. Chem., Int. Ed., 2010, 49, $4825-4828$.

11 A. Manna, P. L. Chen, H. Akiyama, T. X. Wei, K. Tamada and W. Knoll, Chem. Mater., 2003, 15, 20-28.

12 R. Klajn, K. J. M. Bishop and B. A. Grzybowski, Proc. Natl. Acad. Sci. U. S. A., 2007, 104, 10305-10309.

13 A. Kohntopp, A. Dabrowski, M. Malicki and F. Temps, Chem. Commun., 2014, 50, 10105-10107.

14 R. Klajn, J. F. Stoddart and B. A. Grzybowski, Chem. Soc. Rev., 2010, 39, 2203-2237.

15 H. Han, J. Y. Lee and X. M. Lu, Chem. Commun., 2013, 49, 6122-6124.

16 J. H. Schenkel, A. Samanta and B. J. Ravoo, Adv. Mater., 2014, 26, 1076-1080.

17 S. Das, P. Ranjan, P. S. Maiti, G. Singh, G. Leitus and R. Klajn, Adv. Mater., 2013, 25, 422-426.

18 O. Chovnik, R. Balgley, J. R. Goldman and R. Klajn, J. Am. Chem. Soc., 2012, 134, 19564-19567.

19 C. Stoffelen, J. Voskuhl, P. Jonkheijm and J. Huskens, Angew. Chem., Int. Ed., 2014, 53, 3400-3404.

20 D. B. Liu, W. W. Chen, K. Sun, K. Deng, W. Zhang, Z. Wang and X. Y. Jiang, Angew. Chem., Int. Ed., 2011, 50, 4103-4107.

21 C. D. Gomes, O. Jacquet, C. Villiers, P. Thuery, M. Ephritikhine and T. Cantat, Angew. Chem., Int. Ed., 2012, 51, 187-190.

22 M. North, R. Pasquale and C. Young, Green Chem., 2010, 12, 1514-1539.

23 S. N. Riduan, Y. G. Zhang and J. Y. Ying, Angew. Chem., Int. Ed., 2009, 48, 3322-3325.

24 P. G. Jessop, D. J. Heldebrant, X. W. Li, C. A. Eckert and C. L. Liotta, Nature, 2005, 436, 1102.

25 C. U. Vohwinkel, E. Lecuona, H. Y. Sun, N. Sommer, I. Vadasz, N. S. Chandel and J. I. Sznajder, J. Biol. Chem., 2011, 286, 37067-37076.

26 M. Varughese, S. Patole, A. Shama and J. Whitehall, Pediatr. Pulmonol., 2002, 33, 56-64.
27 K. C. Jie, Y. Yao, X. D. Chi and F. H. Huang, Chem. Commun., 2014, 50, 5503-5505.

28 S. J. Lin and P. Theato, Macromol. Rapid Commun., 2013, 34, 1118-1133.

29 Q. Yan, R. Zhou, C. K. Fu, H. J. Zhang, Y. W. Yin and J. Y. Yuan, Angew. Chem., Int. Ed., 2011, 50, 4923-4927.

30 D. Witt, R. Klajn, P. Barski and B. A. Grzybowski, Curr. Org. Chem., 2004, 8, 1763-1797.

31 R. Klajn, Pure Appl. Chem., 2010, 82, 2247-2279.

32 A. R. Rothrock, R. L. Donkers and M. H. Schoenfisch, J. Am. Chem. Soc., 2005, 127, 9362-9363.

33 S. Zhou, X. Chen, T. Nguyen, A. K. Voice and G. T. Rochelle, ChemSusChem, 2010, 3, 913-918.

34 N. H. Khdary and M. A. Ghanem, J. Mater. Chem., 2012, 22, 12032-12038.

35 S. Kadiwala, A. V. Rayer and A. Henni, Chem. Eng. J., 2012, 179, 262-271.

36 J. Alauzun, A. Mehdi, C. Reye and R. J. P. Corriu, J. Am. Chem. Soc., 2005, 127, 11204-11205.

37 Under our experimental conditions, transformation of the carbamate into a cyclic urea is highly unlikely as it typically requires high $\mathrm{CO}_{2}$ pressures, elevated temperatures, and/or the presence of a catalyst (e.g. ref. 38 and 39).

38 M. Tamura, K. Noro, M. Honda, Y. Nakagawa and K. Tomishige, Green Chem., 2013, 15, 1567-1577.

39 C. Wu, H. Cheng, R. Liu, Q. Wang, Y. Hao, Y. Yu and F. Zhao, Green Chem., 2010, 12, 1811-1816.

40 This conclusion is supported by an experiment in which we incubated AuNPs co-functionalized with $\mathbf{3}$ and $\mathbf{C}_{\mathbf{1 2}} \mathbf{S H}$ under oxygen for an extended period of time. Under oxidative conditions, the presence of free $\mathrm{SH}$ groups on the outer surfaces of NPs would likely cause crosslinking due to disulfide bridge formation. No such crosslinking was observed by DLS or UV-Vis spectroscopy.

41 T. Zdobinsky, P. S. Maiti and R. Klajn, J. Am. Chem. Soc., 2014, 136, 2711-2714.

42 In addition to the increasing NP surface polarity in the nonpolar medium, attractive forces between the NPs could originate from direct interactions (e.g. due to dipole-dipole interactions of the cis-azobenzene dipoles). 